\title{
Familial Confounding of the Association between Maternal Smoking During Pregnancy and ADHD in Offspring
}

\author{
Charlotte Skoglund, M.D. ${ }^{1}$, Qi Chen, M.Sc. ${ }^{2}$, Brian M D'Onofrio, PhD. ${ }^{3}$, Paul Lichtenstein, \\ PhD. ${ }^{2}$, and Henrik Larsson, PhD. ${ }^{2}$ \\ ${ }^{1}$ Department of Clinical Neuroscience, Karolinska Institute, Stockholm, Sweden \\ ${ }^{2}$ Department of Medical Epidemiology and Biostatistics, Karolinska Institute, Stockholm, Sweden \\ ${ }^{3}$ Department of Psychological and Brain Sciences, Indiana University, Bloomington, IN, USA
}

\begin{abstract}
Background-Maternal Smoking During Pregnancy (SDP) has consistently been associated with increased risk of attention deficit hyperactivity disorder (ADHD) in offspring, but recent studies indicate that this association might be due to unmeasured familial confounding.
\end{abstract}

Methods-A total of 813030 individuals born in Sweden between 1992 and 2000 were included in this nationwide population based cohort study. Data on maternal SDP and ADHD diagnosis were obtained from national registers and patients were followed up from the age of 3 to the end of 2009. Hazard Ratios (HRs) were estimated using stratified Cox regression models. Cousin and sibling data were used to control for unmeasured familial confounding.

Results-At the population level maternal SDP predicted ADHD in offspring $\left(\mathrm{HR}_{\text {ModerateSDP}}=1.89 ; \mathrm{HR}_{\mathrm{HighSDP}}=2.50\right)$. This estimate gradually attenuated towards the null when adjusting for measured confounders $\left(\mathrm{HR}_{\text {ModerateSDP }}=1.62 ; \mathrm{HR}_{\mathrm{HighSDP}}=2.04\right)$, unmeasured confounders shared within the extended family (i.e., cousin comparison) $\left(\mathrm{HR}_{\mathrm{ModerateSDP}}=1.45\right.$; $\mathrm{HR}_{\mathrm{HighSDP}}=1.69$ ), and unmeasured confounders within the nuclear family (i.e., sibling comparison) $\left(\mathrm{HR}_{\mathrm{ModerateSDP}}=0.88 ; \mathrm{HR}_{\mathrm{HighSDP}}=0.84\right)$.

Conclusions-Our results suggest that the association between maternal SDP and offspring ADHD are due to unmeasured familial confounding.

\section{Keywords}

Maternal smoking during pregnancy; attention-deficit/hyperactivity disorder; confounding; sibling comparisons

Corresponding author: Charlotte Skoglund, Karolinska Institute, Department of Clinical Neuroscience, Administration Z5:00, SE-171 76 Stockholm, SWEDEN. Telephone: +46-8-123 474 93. Telefax: +46-8-346563. charlotte.skoglund@ sll.se.

Conflict of interest: There were no financial or other conflicts of interest for any of the authors. The lead author takes responsibility for the integrity of the data and the accuracy of the data analysis. 


\section{INTRODUCTION}

Attention-Deficit/Hyperactivity Disorder (ADHD), characterized by impairing symptoms of hyperactivity, impulsivity and inattention, is a highly prevalent neuropsychiatric disorder that persists into adulthood in a sizable number of affected children of both genders (Biederman, 2005). In addition to a strong genetic predisposition to ADHD, environmental factors account for an estimated $10 \%$ to $40 \%$ of the variance in liability to the disorder (Banerjee, Middleton, \& Faraone, 2007). Maternal lifestyle factors during pregnancy, such as stress, alcohol use and cigarette smoking may represent environmental risk factors for ADHD in offspring (Thapar, Cooper, Eyre, \& Langley, 2012), but the mechanisms through which such risks influence ADHD is poorly understood.

Studies have shown that prenatal exposure to maternal Smoking During Pregnancy (SDP) is associated with low birth weight and preterm birth (Cnattingius, 2004) and also suggest plausible biological mechanisms through which SDP may influence brain development, including deleterious effects on neurotransmission, neuronal differentiation, and migration (Dwyer, McQuown, \& Leslie, 2009; Slotkin, Tate, Cousins, \& Seidler, 2006). A robust association between SDP and ADHD in offspring has been observed in previous epidemiological studies, even after adjustment for measured confounders (Banerjee, et al., 2007; Langley, Rice, van den Bree, \& Thapar, 2005; Linnet et al., 2003; Motlagh et al., 2010; Rodriguez \& Bohlin, 2005) suggesting possible causality. Other researchers express great skepticism, however, because of the inability of these studies to rule out unmeasured familial factors (Knopik, 2009; Thapar \& Rutter, 2009). For instance, the heritability of both SDP (Agrawal et al., 2008; D'Onofrio et al., 2003; Ellingson, Rickert, Lichtenstein, Langstrom, \& D'Onofrio, 2012) and ADHD (Larsson, Anckarsater, Rastam, Chang, \& Lichtenstein, 2012), as well as the recent reports of a genetic overlap between ADHD and smoking (August et al., 2006; Chang, Lichtenstein, \& Larsson, 2012; Monuteaux et al., 2008), indicate that maternal SDP may reflect a genetic predisposition rather than a causal risk factor for offspring ADHD. Since RCTs are impossible, quasi-experimental designs (e.g., sibling and cousin comparison analyses) are needed to rule out unmeasured familial factors (Rutter, 2009).

Available evidence from studies using different quasi-experimental designs including one study of SDP in both mothers and fathers (Langley, Heron, Smith, \& Thapar, 2012), one in vitro cross-fostering study (Thapar et al., 2009) and three sibling comparison studies (D'Onofrio et al., 2008; Lindblad \& Hjern, 2010; Obel et al., 2011) suggest that the dose dependent relationship between SDP and offspring ADHD is due to unmeasured familial factors (i.e., genetic and/or household-level factors). However, these previous quasiexperimental studies of ADHD have noteworthy limitations. First, studies comparing the offspring ADHD risk between maternal and paternal SDP have produced mixed results and are limited by small sample sizes (Langley, et al., 2012; Nomura, Marks, \& Halperin, 2010). Second. using children who remain unrelated to their mothers through assisted conception to study SDP is a novel approach for disentangling genetic confounds from prenatal risks, but the findings for SDP and ADHD need to be replicated, as the results were based on parentrated ADHD symptoms during childhood from a small study of children conceived through assisted reproductive technologies (Thapar et al., 2009). Third, previous sibling studies have 
been limited by imprecise risk estimates (because of small sample sizes) and by inadequate proxy measures, including use of ADHD medication during only one calendar year (Lindblad \& Hjern, 2010) and maternal-ratings of ADHD related problems (D'Onofrio, et al., 2008). In addition, previous sibling studies were not able to identify and exclude halfsiblings which mean that the effect of familial confounding may have been underestimated (D'Onofrio, et al., 2008; Obel, et al., 2011). Clearly, additional quasi-experimental research is needed to address these limitations.

Using a large population-based cohort of children, we tested the hypothesis that maternal SDP is a causal risk factor for offspring ADHD. To explicitly address concerns about the generalizability of the findings from previous sibling-comparison studies (Talati \& Weissman, 2010) and limitations inherent in the design (e.g., women who vary in their smoking status are different from those that smoke across pregnancies) (D'Onofrio, Lahey, Turkheimer, \& Lichtenstein in press) the current study also included cousin comparisons. We explored the impact of confounding factors by (a) controlling for measured covariates, (b) comparing full-cousins (offspring of adult full-siblings) within extended families to control for all unmeasured factors that make cousins similar and (c) comparing full-siblings within nuclear families to control for all unmeasured factors that make siblings similar.

\section{METHOD}

\section{Sample}

Data sources-We utilized data from a record linkage of eight population-based registries in Sweden; personal identification numbers enabled accurate linkage.

The population-based Medical Birth Register (MBR) provides information on SDP in Sweden since 1983 through self-reported prospective information at the registration to antenatal care (in pregnancy week 8-12). Swedish maternal healthcare has a national homogenous structure and reaches almost $99 \%$ of all Swedish women before pregnancy week 15 (Lindmark \& Cnattingius, 1991). The Patient Register (PR), provide data on psychiatric in-patient care since 1987 (ICD-9 to ICD-10) and out-patient care (ICD-10) since 2001. The $S$ wedish Prescribed Drug register (PDR) (Wettermark et al., 2007) contain information on drug identity (Anatomical Therapeutic Chemical [ATC-codes]) and dates of all registered prescriptions to the entire population in Sweden since July 2005. The MultiGeneration Register (MGR), contain information on the identity of the parents of all residents born in Sweden since 1932. The Cause of Death Register(CDR) provides information on dates of all registered deaths since 1958. The Migration Register (MR) include information on dates of all registered migrations into or out of Sweden since 1969.The LISA Register contain information on education, employment and income of individuals in each household in Sweden at the end of 2009. The Stockholm Children and Adolescents Psychiatric Care Register (Pastill) cover information on psychiatric diagnoses based on both ICD-10 and Diagnostic and Statistical Manual of Mental Disorders (DSM-IV) for all children and adolescents living in Stockholm County since 2001. The study was approved by the research ethics committee at Karolinska Institute, Stockholm, Sweden Protocol nr 2009/5:9. 
Study population-After identification of all individuals born in Sweden between 1992 and $2000(\mathrm{~N}=894444)$ and exclusion of individuals with serious congenital malformations ( $\mathrm{N}=32$ 209), multiple births ( $\mathrm{N}=25302)$, still born before or during delivery $(\mathrm{N}=2767)$, dead before 3 years of age or before 2001( $\mathrm{N}=2$ 613), emigrated before 3 years of age or before $2001(\mathrm{~N}=18226)$, missing data on mother's identification number $(\mathrm{n}=250)$ or who had received an ADHD diagnosis before 3 years of age $(n=47)$ an eligible sample of 813030 individuals (90.90\% of the targeted population) was identified from MBR (Figure 1). After exclusion due to missing values on SDP $(\mathrm{N}=44$ 803) the study population included 768227 individuals covering 365442 full siblings nested within 172701 families and 155852 cousins nested within 52183 families. All the individuals were followed up from the age of 3 until diagnosis of ADHD, death, emigration, or December 31, 2009, whichever occurred first.

\section{Measures}

Offspring Outcomes-We identified 13996 patients with an in- or outpatient diagnosis of Hyperkinetic Disorder (HKD) (F90 in ICD-10) between January 2001 and December 2009 from PR, and 3352 patients with diagnoses of HKD (F90 in ICD-10) or ADHD (DSM-IV:314) from Pastill. A total of 16216 patients treated with stimulant or nonstimulant medication for ADHD (methylphenidate [N06BA04]; atomoxetin [N06BA09]; amphetamine [N06BA01]; dexamphetamine [N06BA02]) at any time between July 2005 and December 2009 were identified via the PDR. Altogether 19891 unique ADHD cases were identified after adjustment of overlaps between the three registers. According to Swedish national clinical guidelines, issued by The National Board of Health and Welfare, ADHD or HKD should be diagnosed by a specialist psychiatrist after a clinical somatic and psychiatric evaluation including childhood history, somatic evaluation and cognitive assessments by a child psychologist. ADHD and HKD diagnoses in PR as well as in Pastill are based on DSM-IV and/or ICD-10 criteria. We used data from 19150 twins (born between 1992 and 2001) with psychiatric symptom information from the Swedish Twin Registry to check the validity of the register-based ADHD diagnosis. ADHD symptoms were assessed using a well-validated measure of 96 specific child psychiatric symptoms (Hansson et al., 2005; Larson et al., 2010). About 70\% of the twins with a national registerbased ADHD diagnosis recorded in the National Patient Register were also rated as screenpositive by parents (Larsson et al., 2013). Because it is well established that discrepancies often exist among different raters (e.g., parents, children, physicians, psychologists) (De Los Reyes \& Kazdin, 2005), the high overlap between different informants suggest that the national register-based diagnoses of ADHD are valid.

Exposure-SDP was measured on a three-point scale (No SDP $=0$, moderate SDP $=1-9$, or high SDP $=\geq 10$ cigarettes per day). The measure has been validated using numerous outcome measures, and has shown to be highly correlated with serum cotinine levels (Lindqvist, Lendahls, Tollbom, Aberg, \& Hakansson, 2002). Previous studies have shown that the validity of self-reported smoking has not changed over time and also acceptable validity of self-reported smoking habits when compared to biochemical markers (measured levels of cotinine in blood) (Hatziandreu et al., 1989). 
Covariates-Based on previous research (D'Onofrio, Singh, Iliadou, Lambe, Hultman, Grann, et al., 2010; D'Onofrio, Singh, Iliadou, Lambe, Hultman, Neiderhiser, et al., 2010; Lundberg et al., 2010), measured covariates included sex, birth year (1992-1994, 19951997 , and 1998-2000), mother's parity $\left(1^{\text {st }}, 2^{\text {nd }}, 3^{\text {rd }}\right.$, or $\left.\geq 4^{\text {th }}\right)$, maternal age at child birth ( $\leq 9,20-24,25-29,30-34$, or $\geq 35$ years), cohabitation with offspring's father (yes or no), maternal highest education ( $\$$ years, $10-12$ years or graduation education) and mother's country of birth (Sweden, other Scandinavian countries or others). Since low birth weight might be an intermediate factor of the association between SDP and ADHD, we choose not to adjust for that measure. Pre-pregnancy BMI is considered partly adjusted for by maternal education level and could potentially be an intermediate factor, thus not included as a covariate in the model.

\section{Statistical Analyses}

We used Cox proportional survival analysis to estimate the magnitude of the associations between SDP and offspring ADHD at the population level. The models calculated Hazard ratios (HRs) for time to ADHD diagnosis. Robust standard errors adjusted the 95\% confidence intervals (CIs) for the presence of familial clustering in the analyses at the population level. In addition to a crude model, we also adjusted for the above mentioned measured covariates.

To explore the effect of unmeasured familial confounding we applied stratified Cox regression models to cousin and sibling data with a separate stratum for each set of full cousins and siblings, respectively. In the cousin sample, 41028 cousins nested in 13372 extended families were discordantly exposed to SDP, while in the sibling sample, there were 26826 discordantly exposed siblings nested in 12507 nuclear families. Cousin comparisons adjust for all unmeasured factors that are constant within the extended family, while the sibling comparisons adjust for factors that are constant within the nuclear family. The stratified Cox regression models using cousins were adjusted for the same covariates as in the models at the population level. The regression models applied to siblings were not adjusted for maternal highest education and mother's country of birth, as these were regarded constant or unlikely to change within siblings.

We conducted sensitivity analyses to assess the potential modifying effect of birth order on the studied associations. We also analyzed a birth year restricted sibling cohort to explore potential bias from outcome misclassification. Furthermore, we explored the generalizability of our results by comparing the sibling sample to the entire cohort (D'Onofrio et al., in press). All statistical analyses were conducted in SAS software version 9.3 (SAS Institute, Cary, NC, USA).

\section{RESULTS}

Table 1 shows the distribution of offspring and maternal covariates. Offspring exposed to maternal SDP were more likely to be of late parity $(\mathrm{p}<.0001)$, have mothers with lower education $(p<.01)$, and their mothers were less likely to cohabit with their biological father at childbirth. 
In Table 2 the crude association showed that offspring exposed to maternal SDP were at increased risk for ADHD ( $\left.\mathrm{HR}_{\text {ModerateSDP }} 1.89 ; \mathrm{HR}_{\mathrm{HighSDP}} 2.50\right)$. This dose-dependent association decreased marginally after adjustment for measured covariates and remained statistically significant ( $\left.\mathrm{HR}_{\text {ModerateSDP }} 1.62 ; \mathrm{HR}_{\mathrm{HighSDP}} 2.04\right)$. The associations were further attenuated in the cousin comparisons; that is, after adjustment for all unmeasured factors that are constant within extended families ( $\left.\mathrm{HR}_{\text {ModerateSDP }} 1.45 ; \mathrm{HR}_{\mathrm{HighSDP}} 1.69\right)$. Sibling comparisons showed that the associations observed at the population level were completely attenuated and no longer statistically significant ( $\mathrm{HR}_{\text {ModerateSDP }} 0.88 ; \mathrm{HR}_{\mathrm{HighSDP}} 0.84$ ), indicating that unmeasured familial factors that are constant within nuclear families explain the associations.

\section{Sensitivity analyses}

Sensitivity analyses were conducted to explore assumptions and to test the robustness of the findings.

Second-born offspring were more often exposed to maternal SDP than first-born offspring. Therefore, we used another sibling sample including only first- and second-born offspring $(\mathrm{N}=345402)$ from each nuclear family to test the modifying effect of birth order on the studied association. Stratified analyses at the population level showed similar results for first-born and second-born siblings (HR ModerateSDP_FirstBorn $_{1.62}$ 95\%CI, 1.49-1.76 $\mathrm{HR}_{\text {HighSDP_FirstBorn }} 1.95$ 95\%CI, 1.76-2.16 HR ModerateSDP_SecondBorn $_{1.58} 95 \%$ CI, $1.42-$ 1.75 and HR $_{\text {HighSDP_SecondBorn }} 2.1395 \% \mathrm{CI}, 1.90-2.39$ ).

Because the outpatient register (started in 2001) and PDR (started in 2005) are relatively new, we explored potential bias from outcome misclassification. For instance, older siblings with an ADHD diagnosis early in life may not have been recorded with a diagnosis in this register. To minimize this potential bias, we performed a separate sibling comparison within a restricted cohort of offspring born 1994 to 1998. The two siblings from each nuclear family that were closest in age were selected for sibling comparisons, resulting in 123974 full siblings nested within 61987 families (the average age difference between siblings was 2.34 years). The results from the restricted sibling sample ( $\mathrm{HR}_{\text {ModerateSDP }}, 1.01 ; 95 \% \mathrm{CI}$, $\left.0.73-1.41 ; \mathrm{HR}_{\text {HighSDP }} 1.08 ; 95 \% \mathrm{CI}, 0.70-1.66\right)$ were similar to that observed in the full sibling sample.

Finally, we explored the generalizability of the results from siblings to the entire cohort. We analyzed Siblings as unrelated individuals using robust standard errors and the results in the sibling sample (HR ModerateSDP $_{1.62}$; 95\%CI, 1.51-1.73; HR HighSDP 2.06 ; 95\%CI, 1.902.22) were very similar to that in the entire cohort ( $\mathrm{HR}_{\text {ModerateSDP }}, 1.62 ; 95 \% \mathrm{CI}, 1.56-1.69$; $\mathrm{HR}_{\text {HighSDP }} 2.04$; 95\% CI, 1.95-2.13).

\section{DISCUSSION}

In this population based cohort study, we used two different quasi-experimental designs (i.e., cousin and sibling comparisons) to explore the mechanisms through which maternal SDP influence ADHD. Our results suggest that the association between maternal SDP and offspring ADHD can be ascribed to unmeasured familial confounding. Similar results have 
been reported in quasi-experimental studies of ADHD from Finland (Obel, et al., 2011), UK (Langley, et al., 2012; Thapar, et al., 2009) and the US (D'Onofrio, et al., 2008), indicating that the study results generalize across samples with different demographic, racial, or ethnic characteristics. These results are also consistent with quasi-experimental studies of SDP for ADHD related outcomes, such as disruptive behaviours, criminality, and academic/cognitive problems (D'Onofrio, et al., in press).

Our study addresses four critical limitations of previous sibling comparison studies (D'Onofrio, et al., 2008; Lindblad \& Hjern, 2010; Obel, et al., 2011). First, our study was based on more than twice as many ADHD cases compared to the previous register-based sibling studies (Lindblad \& Hjern, 2010; Obel, et al., 2011), which allowed for more precise estimates (i.e., narrow confidence intervals) of the magnitude of the associations between SDP and offspring ADHD, even in the sibling and cousin comparisons. Second, the prior Swedish register-based study identified ADHD cases from medication use during one calendar year. This may have introduced bias in the sibling analyses due to misclassification of discordant and concordant sibling pairs. The large difference in the number of identified ADHD cases (despite similar $\mathrm{N}$ for the study population) between studies, coupled with sensitivity analyses based on a restricted cohort of siblings close in age, indicate that bias from outcome misclassification is unlikely in our study. Third, previous studies might be confounded by the fact that mothers reported on both SDP and offspring behaviors (D'Onofrio, et al., 2008), while we in contrast used measured SDP via self-ratings at the first registration to antenatal care and obtained outcome information via clinical diagnosis made by physicians.

Finally, the results from previous sibling studies have been questioned by researchers who have explicitly hypothesized that women who vary in their smoking status across pregnancies are not comparable to all smoking women (Frisell, Oberg, Kuja-Halkola, \& Sjolander, 2012). We used cousin comparisons to overcome the concern of external validity and observed converging evidence across different quasi-experimental designs, which further strengthen the familial confounding hypothesis.

Our results should be interpreted in the context of some limitations. Although sibling comparison will not be confounded by factors shared by siblings, the estimates might theoretically be more sensitive to bias due to non-shared confounders than the unpaired estimates (Frisell, et al., 2012). The ascertainment of ADHD cases was predominantly based on ICD-10 diagnosis of hyperkinetic disorder and prescribed medication unique for the treatment of ADHD. The ICD-10 definition of ADHD is stricter compared with that in DSM-IV, and National guidelines for medication of ADHD, issued by the Swedish National Board of health and Welfare in 2002, stated that medication should be reserved for cases where other supportive interventions have failed, indicating that our proxies for ADHD most likely underestimates the incidence of ADHD and identifies severe ADHD cases. Thus, our strategies probably could not avoid producing false negatives, while we consider bias due to false positives more unlikely. It should also be noted that our findings do not rule out the possibility of an association between SDP and more refined ADHD-related neurocognitive deficits, such as executive function, response inhibition, and fine motor skills. To further explore this issue, studies using more detailed and refined outcome measures are warranted. 
As in all observational studies, we could not fully rule out residual confounding due to a lack of intact information on the exposure variable and other potential confounders. Also, as SDP was assessed at the first visit to antenatal care and used as a proxy for the entire pregnancy, there is a possibility of misclassification of exposure. In addition, pregnant women may also conceal their smoking habits (Lindqvist, et al., 2002). However, previous studies have repeatedly shown support for a causal association between maternal SDP and low birth weight suggesting that the effect of exposure misclassification is small in magnitude (Cnattingius, 2004; D'Onofrio et al., 2012).

\section{CONCLUSION}

In conclusion, our data suggest that the previously observed association between maternal SDP and ADHD can be ascribed to unmeasured familial confounding. There is mounting evidence that SDP is harmful in many ways (e.g., low birth weight and infant mortality) and our results should not be interpreted as an argument for changing the recommendations that women should not smoke during pregnancy. Nevertheless, we view our study as a contribution to the current state of knowledge concerning causal risk factors for ADHD. It is essential for clinicians, researchers and policy makers to focus on true causal risk factors and SDP is most probably not one of these.

\section{Acknowledgments}

Financial support was provided through the regional agreement on medical training and clinical research (K1426-2011) between Stockholm County Council and Karolinska Institute; and through grants from the Swedish Council for Working Life and Social Research, and the Swedish Research Council (2010-3184; 2011-2492).

\section{Abbreviations}

$\begin{array}{ll}\text { SDP } & \text { Maternal Smoking During Pregnancy } \\ \text { ADHD } & \text { attention deficit hyperactivity disorder } \\ \text { HKD } & \text { Hyperkinetic disorder } \\ \text { MBR } & \text { Medical Birth Register } \\ \text { PR } & \text { Patient Register } \\ \text { PDR } & \text { Swedish Prescribed Drug register } \\ \text { MGR } & \text { Multi-Generation Register } \\ \text { CDR } & \text { Cause of Death Register } \\ \text { MR } & \text { Migration Register } \\ \text { Pastill } & \text { Stockholm Children and Adolescents Psychiatric Care Register } \\ \text { ATC-codes } & \text { Anatomical Therapeutic Chemical Codes } \\ \text { DSM-IV } & \text { Diagnostic and Statistical Manual of Mental Disorders }\end{array}$




\section{REFERENCES}

Agrawal A, Knopik VS, Pergadia ML, Waldron M, Bucholz KK, Martin NG, et al. Correlates of cigarette smoking during pregnancy and its genetic and environmental overlap with nicotine dependence. Nicotine Tob Res. 2008; 10(4):567-578. [PubMed: 18418779]

August GJ, Winters KC, Realmuto GM, Fahnhorst T, Botzet A, Lee S. Prospective study of adolescent drug use among community samples of ADHD and non-ADHD participants. J Am Acad Child Adolesc Psychiatry. 2006; 45(7):824-832. [PubMed: 16832319]

Banerjee TD, Middleton F, Faraone SV. Environmental risk factors for attention-deficit hyperactivity disorder. Acta Paediatr. 2007; 96(9):1269-1274. [PubMed: 17718779]

Biederman J. Attention-deficit/hyperactivity disorder: a selective overview. Biol Psychiatry. 2005; 57(11):1215-1220. [Review]. [PubMed: 15949990]

Chang Z, Lichtenstein P, Larsson H. The effects of childhood ADHD symptoms on early-onset substance use: a Swedish twin study. J Abnorm Child Psychol. 2012; 40(3):425-435. [PubMed: 21947618]

Cnattingius S. The epidemiology of smoking during pregnancy: smoking prevalence, maternal characteristics, and pregnancy outcomes. Nicotine Tob Res. 2004; 6(Suppl 2):S125-S140. [PubMed: 15203816]

D'Onofrio BM, Rickert ME, Langstrom N, Donahue KL, Coyne CA, Larsson H, et al. Familial confounding of the association between maternal smoking during pregnancy and offspring substance use and problems. Arch Gen Psychiatry. 2012; 69(11):1140-1150. [PubMed: 23117635]

D'Onofrio BM, Singh AL, Iliadou A, Lambe M, Hultman CM, Grann M, et al. Familial confounding of the association between maternal smoking during pregnancy and offspring criminality: a population-based study in Sweden. Arch Gen Psychiatry. 2010; 67(5):529-538. [PubMed: 20439834]

D'Onofrio BM, Singh AL, Iliadou A, Lambe M, Hultman CM, Neiderhiser JM, et al. A quasiexperimental study of maternal smoking during pregnancy and offspring academic achievement. Child Dev. 2010; 81(1):80-100. [PubMed: 20331655]

D'Onofrio BM, Turkheimer EN, Eaves LJ, Corey LA, Berg K, Solaas MH, et al. The role of the children of twins design in elucidating causal relations between parent characteristics and child outcomes. J Child Psychol Psychiatry. 2003; 44(8):1130-1144. [PubMed: 14626455]

D'Onofrio BM, Van Hulle CA, Waldman ID, Rodgers JL, Harden KP, Rathouz PJ, et al. Smoking during pregnancy and offspring externalizing problems: an exploration of genetic and environmental confounds. Dev Psychopathol. 2008; 20(1):139-164. [PubMed: 18211732]

D'Onofrio BM, Lahey BB, Turkheimer E, Lichtenstein P. The critical need for family-based, quasiexperimental research in integrating genetic and social science research. American Journal of Public Health. ((in press)).

De Los Reyes A, Kazdin AE. Informant discrepancies in the assessment of childhood psychopathology: a critical review, theoretical framework, and recommendations for further study. Psychol Bull. 2005; 131(4):483-509. [PubMed: 16060799]

Dwyer JB, McQuown SC, Leslie FM. The dynamic effects of nicotine on the developing brain. Pharmacol Ther. 2009; 122(2):125-139. [PubMed: 19268688]

Ellingson JM, Rickert ME, Lichtenstein P, Langstrom N, D'Onofrio BM. Disentangling the relationships between maternal smoking during pregnancy and co-occurring risk factors. Psychol Med. 2012; 42(7):1547-1557. [PubMed: 22115276]

Frisell T, Oberg S, Kuja-Halkola R, Sjolander A. Sibling comparison designs: bias from non-shared confounders and measurement error. Epidemiology. 2012; 23(5):713-720. [PubMed: 22781362]

Hansson SL, Svanstrom Rojvall A, Rastam M, Gillberg C, Gillberg C, Anckarsater H. Psychiatric telephone interview with parents for screening of childhood autism - tics, attention-deficit hyperactivity disorder and other comorbidities (A-TAC): preliminary reliability and validity. Br J Psychiatry. 2005; 187:262-267. [PubMed: 16135864]

Hatziandreu EJ, Pierce JP, Fiore MC, Grise V, Novotny TE, Davis RM. The reliability of self-reported cigarette consumption in the United States. Am J Public Health. 1989; 79(8):1020-1023.

[PubMed: 2751017] 
Knopik VS. Maternal smoking during pregnancy and child outcomes: real or spurious effect? Dev Neuropsychol. 2009; 34(1):1-36. [PubMed: 19142764]

Langley K, Heron J, Smith GD, Thapar A. Maternal and paternal smoking during pregnancy and risk of ADHD symptoms in offspring: testing for intrauterine effects. Am J Epidemiol. 2012; 176(3): 261-268. [PubMed: 22791738]

Langley K, Rice F, van den Bree MB, Thapar A. Maternal smoking during pregnancy as an environmental risk factor for attention deficit hyperactivity disorder behaviour. A review. Minerva Pediatr. 2005; 57(6):359-371. [PubMed: 16402008]

Larson T, Anckarsater H, Gillberg C, Stahlberg O, Carlstrom E, Kadesjo B, et al. The autism--tics, $\mathrm{AD} / \mathrm{HD}$ and other comorbidities inventory (A-TAC): further validation of a telephone interview for epidemiological research. BMC Psychiatry. 2010; 10:1. [PubMed: 20055988]

Larsson H, Anckarsater H, Rastam M, Chang Z, Lichtenstein P. Childhood attention-deficit hyperactivity disorder as an extreme of a continuous trait: a quantitative genetic study of 8,500 twin pairs. J Child Psychol Psychiatry. 2012; 53(1):73-80. [PubMed: 21923806]

Larsson H, Ryden E, Boman M, Langstrom N, Lichtenstein P, Landen M. Risk of bipolar disorder and schizophrenia in relatives of people with attention-deficit hyperactivity disorder. Br J Psychiatry. 2013

Lindblad F, Hjern A. ADHD after fetal exposure to maternal smoking. Nicotine Tob Res. 2010; 12(4): 408-415. [PubMed: 20176681]

Lindmark G, Cnattingius S. The scientific basis of antenatal care. Report from a state-of-the-art conference. Acta Obstet Gynecol Scand. 1991; 70(2):105-109. [PubMed: 1679279]

Lindqvist R, Lendahls L, Tollbom O, Aberg H, Hakansson A. Smoking during pregnancy: comparison of self-reports and cotinine levels in 496 women. Acta Obstet Gynecol Scand. 2002; 81(3):240 244. [PubMed: 11966481]

Linnet KM, Dalsgaard S, Obel C, Wisborg K, Henriksen TB, Rodriguez A, et al. Maternal lifestyle factors in pregnancy risk of attention deficit hyperactivity disorder and associated behaviors: review of the current evidence. Am J Psychiatry. 2003; 160(6):1028-1040. [PubMed: 12777257]

Lundberg F, Cnattingius S, D'Onofrio B, Altman D, Lambe M, Hultman C, et al. Maternal smoking during pregnancy and intellectual performance in young adult Swedish male offspring. Paediatr Perinat Epidemiol. 2010; 24(1):79-87. [PubMed: 20078833]

Monuteaux MC, Faraone SV, Hammerness P, Wilens TE, Fraire M, Biederman J. The familial association between cigarette smoking and ADHD: a study of clinically referred girls with and without ADHD, and their families. Nicotine Tob Res. 2008; 10(10):1549-1558. [PubMed: 18946774]

Motlagh MG, Katsovich L, Thompson N, Lin H, Kim YS, Scahill L, et al. Severe psychosocial stress and heavy cigarette smoking during pregnancy: an examination of the pre- and perinatal risk factors associated with ADHD and Tourette syndrome. Eur Child Adolesc Psychiatry. 2010; 19(10):755-764. [PubMed: 20532931]

Nomura Y, Marks DJ, Halperin JM. Prenatal exposure to maternal and paternal smoking on attention deficit hyperactivity disorders symptoms and diagnosis in offspring. J Nerv Ment Dis. 2010; 198(9):672-678. [PubMed: 20823730]

Obel C, Olsen J, Henriksen TB, Rodriguez A, Jarvelin MR, Moilanen I, et al. Is maternal smoking during pregnancy a risk factor for hyperkinetic disorder?--Findings from a sibling design. Int $\mathbf{J}$ Epidemiol. 2011; 40(2):338-345. [Research Support, Non-U.S. Gov't]. [PubMed: 21075808]

Rodriguez A, Bohlin G. Are maternal smoking and stress during pregnancy related to ADHD symptoms in children? J Child Psychol Psychiatry. 2005; 46(3):246-254. [PubMed: 15755301]

Rutter M. Understanding and testing risk mechanisms for mental disorders. J Child Psychol Psychiatry. 2009; 50(1-2):44-52. [PubMed: 19220588]

Slotkin TA, Tate CA, Cousins MM, Seidler FJ. Prenatal nicotine exposure alters the responses to subsequent nicotine administration and withdrawal in adolescence: Serotonin receptors and cell signaling. Neuropsychopharmacology. 2006; 31(11):2462-2475. [PubMed: 16341021]

Talati A, Weissman MM. In utero smoking exposure warrants further investigation. Arch Gen Psychiatry. 2010; 67(10):1094. [PubMed: 20921125] 
Thapar A, Cooper M, Eyre O, Langley K. Practitioner Review: What have we learnt about the causes of ADHD? J Child Psychol Psychiatry. 2012

Thapar A, Rice F, Hay D, Boivin J, Langley K, van den Bree M, et al. Prenatal smoking might not cause attention-deficit/hyperactivity disorder: evidence from a novel design. Biol Psychiatry. 2009; 66(8):722-727. [PubMed: 19596120]

Thapar A, Rutter M. Do prenatal risk factors cause psychiatric disorder? Be wary of causal claims. Br J Psychiatry. 2009; 195(2):100-101. [PubMed: 19648537]

Wettermark B, Hammar N, Fored CM, Leimanis A, Otterblad Olausson P, Bergman U, et al. The new Swedish Prescribed Drug Register--opportunities for pharmacoepidemiological research and experience from the first six months. Pharmacoepidemiol Drug Saf. 2007; 16(7):726-735. [PubMed: 16897791] 


\section{Key Points}

- Maternal smoking during pregnancy is commonly cited in the literature as a risk factor for ADHD in offspring.

- In this nation-wide register based cohort study cousin and sibling data were used to control for unmeasured familial confounding.

- Our results suggest that the association between maternal smoking during pregnancy and offspring ADHD can be ascribed to unmeasured familial confounding.

- It is essential for clinicians, researchers and policy makers to focus on true causal risk factors and maternal smoking during pregnancy is most probably not one of these. 


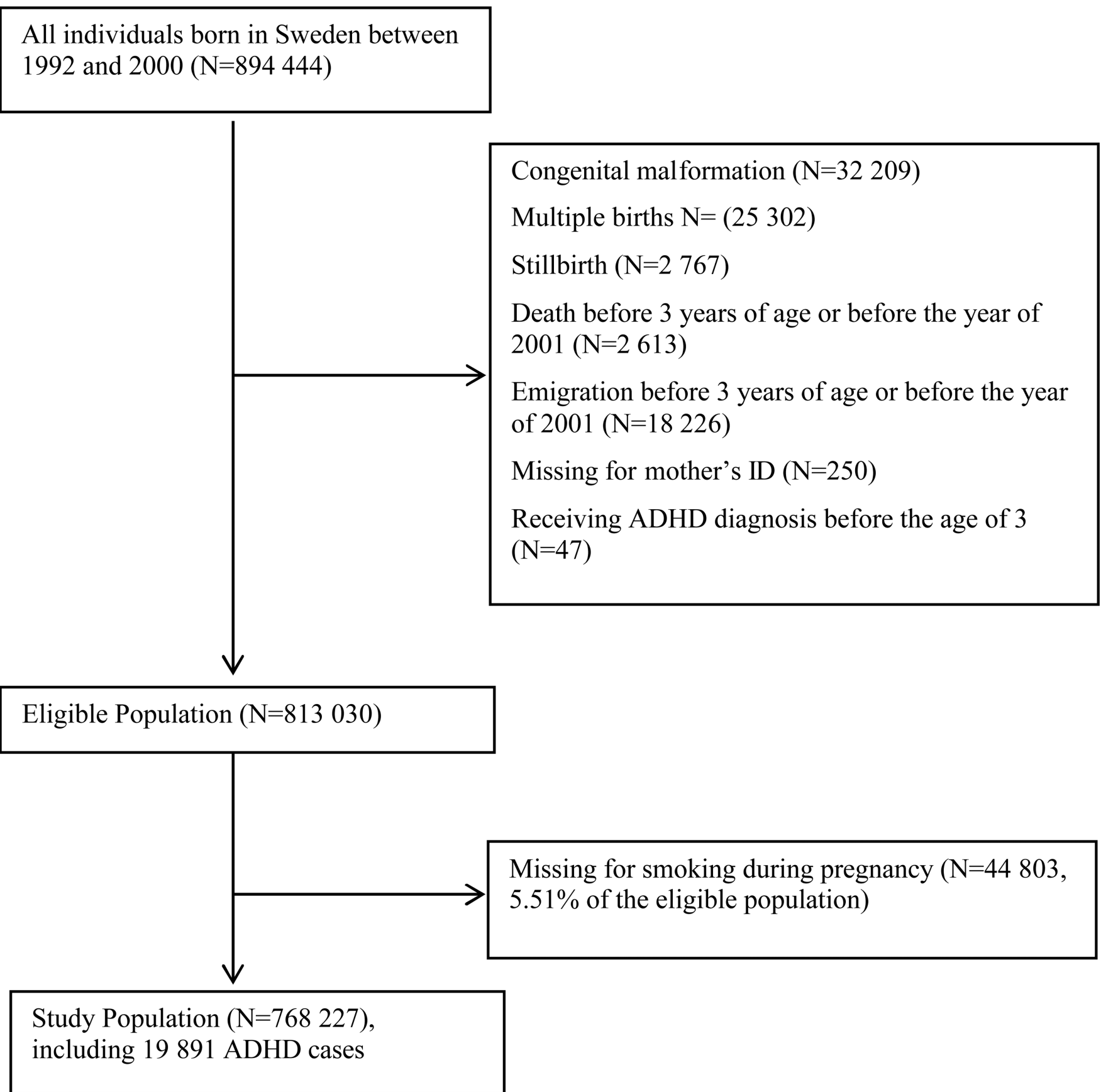

Figure 1.

Sample selection 


\section{Table 1}

Demographic characteristics of mothers and offspring

\begin{tabular}{|c|c|c|c|}
\hline & $\begin{array}{l}\text { Entire cohort } \\
(\mathrm{N}=768227)\end{array}$ & $\begin{array}{l}\text { Cousins } b \\
(\mathrm{~N}=155852)\end{array}$ & $\begin{array}{l}\begin{array}{l}\text { Full siblings } c \\
(\mathrm{~N}=365 \text { 442) }\end{array} \\
\end{array}$ \\
\hline Covariates & {$[\mathrm{N}(\%)]$} & {$[\mathrm{N}(\%)]$} & {$[\mathrm{N}(\%)]$} \\
\hline \multicolumn{4}{|l|}{ SDP } \\
\hline No cigarette & $638400(83.1)$ & $129362(83.0)$ & $317836(87.0)$ \\
\hline 1-9 cigarettes per day & $83830(10.9)$ & $16991(10.9)$ & $31321(8.6)$ \\
\hline$\geq 10$ cigarettes per day & $45997(6.0)$ & $9499(6.1)$ & $16282(4.5)$ \\
\hline \multicolumn{4}{|l|}{ Sex } \\
\hline Male & $392865(51.1)$ & $79688(51.1)$ & $187460(51.3)$ \\
\hline Female & $375282(48.9)$ & $76164(48.9)$ & $177982(48.7)$ \\
\hline \multicolumn{4}{|l|}{ Birth year } \\
\hline 1992-1994 & $301273(39.2)$ & $60917(39.1)$ & $124104(34.0)$ \\
\hline 1995-1997 & $247989(32.3)$ & $51532(33.1)$ & $142840(39.1)$ \\
\hline 1998-2000 & $218965(28.5)$ & $43403(27.8)$ & $98498(27.0)$ \\
\hline \multicolumn{4}{|l|}{ Mother's parity } \\
\hline $1^{\text {st }}$ & $314257(40.9)$ & $60719(39.0)$ & $133153(36.4)$ \\
\hline $2^{\text {nd }}$ & $284924(37.1)$ & $59325(38.1)$ & $157804(43.2)$ \\
\hline $3^{\text {rd }}$ & $116842(15.2)$ & $25300(16.2)$ & $50570(13.8)$ \\
\hline$\geq^{\text {th }}$ & $52187(6.8)$ & $10508(6.7)$ & $23915(6.5)$ \\
\hline \multicolumn{4}{|l|}{ Maternal age at child birth (years) } \\
\hline$\leq 9$ & $11282(1.5)$ & $1752(1.1)$ & $3621(0.99)$ \\
\hline $20-24$ & 120223 (15.6) & $23964(15.4)$ & $60434(16.5)$ \\
\hline 25-29 & $278200(36.2)$ & $60992(39.1)$ & $144326(39.5)$ \\
\hline 30-34 & $238729(31.1)$ & $50053(32.1)$ & $113786(31.0)$ \\
\hline $\mathbf{3 5}$ & $119793(15.6)$ & $19091(12.3)$ & $43275(11.8)$ \\
\hline \multicolumn{4}{|l|}{ Cohabit with offspring's father } \\
\hline Yes & $697508(94.9)$ & $148971(95.6)$ & $355678(97.3)$ \\
\hline No & $37309(5.1)$ & $6881(4.4)$ & $9768(2.7)$ \\
\hline \multicolumn{4}{|l|}{ Maternal highest education } \\
\hline$\$ 9$ years & $73484(9.8)$ & $12440(8.0)$ & $31136(8.7)$ \\
\hline 10-12 years & $381133(50.6)$ & $81879(52.5)$ & $178615(49.8)$ \\
\hline Graduate education & $298949(39.7)$ & $61533(39.5)$ & $149295(41.6)$ \\
\hline \multicolumn{4}{|l|}{ Mother's country of birth } \\
\hline Sweden & $647746(84.3)$ & $148511(95.3)$ & $310254(84.9)$ \\
\hline Denmark. Finland. Norway. or Iceland & $20626(2.7)$ & $2603(1.7)$ & $8332(2.3)$ \\
\hline Other & $99837(13.0)$ & $4738(3.0)$ & $46851(12.8)$ \\
\hline
\end{tabular}


${ }^{c}$ In the full sibling sample, 6396 individuals were missing data on education and 5 for mother's country of birth. 
Table 2

Relative risks of ADHD among offspring exposed to smoking during pregnancy

\begin{tabular}{|c|c|c|c|c|}
\hline \multirow[b]{2}{*}{ Exposure } & \multicolumn{3}{|c|}{$H R(95 \% C I)$} & \multirow[b]{2}{*}{ Full sibling $d$} \\
\hline & Crude $^{a}$ & Adjusted $^{b}$ & Cousins $c$ & \\
\hline No SDP & Reference & Reference & Reference & Reference \\
\hline Moderate SDP (1-9 cigarettes per day) & $1.89(1.83-1.97)$ & $1.62(1.56-1.69)$ & $1.45(1.24-1.68)$ & $0.88(0.73-1.06)$ \\
\hline High SDP ( $>10$ cigarettes per day) & $2.50(2.40-2.61)$ & $2.04(1.95-2.13)$ & $1.69(1.40-2.04)$ & $0.84(0.65-1.06)$ \\
\hline \multicolumn{5}{|l|}{${ }^{a} \mathrm{~N}=768227$} \\
\hline \multicolumn{5}{|c|}{$\begin{array}{l}b_{\mathrm{N}}=720853 \text { Adjusted for offspring gender, birth year, mother's parity, maternal age, cohabitation status, maternal highest education, and } \\
\text { mother's country of birth. }\end{array}$} \\
\hline
\end{tabular}

